\title{
Retroperitoneal Fibrosis and its Differential Diagnoses: The Role of Radiological Imaging
}

\section{Retroperitoneale Fibrose und ihre Differenzialdiagnosen: Die Rolle der radiologischen Bildgebung}

Authors

Felix Peisen ${ }^{1}$, Wolfgang Maximilian Thaiss ${ }^{1}$, Kaspar Ekert ${ }^{1}$, Marius Horger ${ }^{1}$, Bastian Amend ${ }^{2}$, Jens Bedke ${ }^{2}$, Konstantin Nikolaou', Sascha Kaufmann ${ }^{1}$

Affiliations

1 Department of Diagnostic and Interventional Radiology, Eberhard Karls University Tübingen, Tübingen, Germany

2 Department of Urology, Eberhard Karls University Tübingen, Tübingen, Germany

Key words retroperitoneum, urinary, fibrosis, cystic, inflammation

received 02.01 .2020

accepted 24.04.2020

\section{Bibliography}

DOI https://doi.org/10.1055/a-1181-9205

Published online: 22.7.2020

Fortschr Röntgenstr 2020; 192: 929-936

(c) Georg Thieme Verlag KG, Stuttgart · New York

ISSN 1438-9029

Correspondence

Prof. Dr. med. Sascha Kaufmann

Radiology, Diagnostic and Interventional Radiology, Hoppe-Seyler-Str.3, 72076 Tübingen, Germany

Tel.: ++49/70 71/2986676

sascha.kaufmann@med.uni-tuebingen.de

\section{ABSTRACT}

Background Retroperitoneal fibrosis is a rare disease with an incidence of $0-1 / 100000$ inhabitants per year and is associated with chronic inflammatory fibrosis of the retroperitoneum and the abdominal aorta. This article sheds light on the role of radiological imaging in retroperitoneal fibrosis, names various differential diagnoses and provides an overview of drug and surgical treatment options.

Methods A literature search for the keywords "retroperitoneal fibrosis" and "Ormond's disease" was carried out in the PubMed database between January 1, 1995 and December 31, $2019(n=1806)$. Mainly original papers were selected, but also reviews, in English and German language, with a focus on publications in the last 10 years, without excluding older publications that the authors believe are relevant to the topic discussed in the review $(n=40)$.
Results and Conclusion Ormond's disease is a rare but important differential diagnosis for nonspecific back and flank pain. Imaging diagnostics using CT or MRI show a retroperitoneal mass, which must be differentiated from lymphoma, sarcoma, multiple myeloma and Erdheim-Chester disease. Patients have an excellent prognosis under adequate therapy. FDG-PET/CT or FDG-PET/MRT should be considered as potential modalities, as hybrid imaging can evaluate both the morphological changes and the inflammation.

Key Points:

- Ormond's disease is a differential diagnosis for nonspecific back and flank pain.

- Radiological imaging is essential and the gold standard in the diagnosis and follow-up of RPF.

- Patients have an excellent prognosis under adequate therapy.

\section{Citation Format}

- Peisen F, Thaiss WM, Ekert K et al. Retroperitoneal Fibrosis and its Differential Diagnoses: The Role of Radiological Imaging. Fortschr Röntgenstr 2020; 192: 929-936

\section{ZUSAMMENFASSUNG}

Hintergrund Die retroperitoneale Fibrose ist mit einer Inzidenz von 0-1/100 000 Einwohner/Jahr eine seltene Erkrankung, welche mit einer chronisch-entzündlichen Fibrosierung des Retroperitoneums und der abdominalen Aorta einhergeht. Dieser Artikel beleuchtet die Rolle der radiologischen Bildgebung bei retroperitonealer Fibrose, benennt verschiedene Differenzialdiagnosen und gibt einen Überblick der medikamentösen und operativen Therapieoptionen.

Methoden Es wurde eine Literaturrecherche zu den Stichwörtern „retroperitoneal fibrosis“ und „Ormond's disease“ zwischen dem 01.01.1995 und dem 31.12.2019 in der Datenbank PubMed durchgeführt $(n=1806)$. Selektiert wurden hauptsächlich Originalarbeiten, aber auch Übersichtsarbeiten, in englischer und deutscher Sprache mit Fokus auf Publikationen in den letzten 10 Jahren, ohne jedoch ältere Publikation auszuschließen, die nach Ansicht der Autoren relevant für das im Review diskutierte Thema sind $(n=40)$.

Ergebnisse und Schlussfolgerung Der Morbus Ormond ist eine zwar seltene, aber wichtige Differenzialdiagnose bei un- 
spezifischen Rücken- und Flankenschmerzen. Die bildgebende Diagnostik mittels CT oder MRT zeigt eine retroperitoneale Raumforderung, welche differenzialdiagnostisch insbesondere von Lymphomen, Sarkomen, dem multiplen Myelom sowie der Erdheim-Chester-Erkrankung abgegrenzt werden muss.
Patienten haben unter adäquater Therapie eine ausgezeichnete Prognose. Ggf. kann eine FDG-PET/CT oder FDG-PET/MRT erwogen werden, da die Hybridbildgebung sowohl die morphologischen Veränderungen als auch die Inflammation durch den FDG-Tracer-Uptake bewerten kann.

\section{Introduction}

With an incidence of $0-1 / 100000$ inhabitants per year, Ormond's disease is a rare disease associated with chronic inflammatory fibrosis of the retroperitoneum and the abdominal aorta. The disease peaks in the 5th-6th decade of life [1]. Vogt et al. provide a detailed description of the difficulties regarding terminology in the German language. The term Ormond's disease is often used synonymously with chronic periaortitis which in turn includes idiopathic retroperitoneal fibrosis (IRF), inflammatory retroperitoneal aortic aneurysm (IRAA), and perianeurysmatic retroperitoneal fibrosis (PARF). The term Ormond's disease is often also used specifically for IRF or retroperitoneal fibrosis [2]. In this article, Ormond's disease is described as idiopathic retroperitoneal fibrosis. The symptoms are nonspecific and range from unclear abdominal and flank pain to classic B-symptoms with fever, fatigue, and weight loss. $80-100 \%$ of patients have an elevated level of C-reactive protein [1]. The most common complication of retroperitoneal fibrosis is obstruction of the urinary tract with subsequent hydronephrosis, which can be unilateral or bilateral. One of the most significant challenges regarding diagnostic imaging is differentiation with respect to other primarily inflammatory and malignant diseases of the retroperitoneum [3]. In $75 \%$ of cases, the etiology cannot be determined so that idiopathic fibrosis (IRF) is diagnosed [4]. In recent years there has been an increasing focus on the theory of IgG4-associated retroperitoneal fibrosis, which is based on the histological verification of IgG4positive plasma cell infiltrates. [5]

\section{Etiology, disease course, and differential diagnoses}

As a rule, a differentiation is made between a primary = idiopathic form (corresponding to Ormond's disease) and a secondary form of retroperitoneal fibrosis. However, a reliable classification is often not possible. Malignant diseases must be ruled out as the cause of RPF.

Initially tissue edema, hypervascularization, and a multitude of mononuclear cells, fibroblasts, and collagen bundles are primarily seen on histopathology. In the late phase, increasing sclerosis and individual calcifications are seen [1]. Acquisition of a pretreatment histopathological specimen is controversial. Authors like Cristian et al. postulate that morphological imaging is not sufficiently accurate to rule out a malignant cause of RPF [6]. Other authors report the opposite and present significant correlations between morphological criteria (extension above the vessels of the renal pedicle, retroperitoneal extension with lifting of the abdominal aorta, additional detection of enlarged lymph nodes) and functional parameters (very high tracer uptake with atypical distribution on FDG-PET/CT) as criteria for a malignant cause of RPF [3, 7,
8]. Therefore, it is unclear whether histopathological confirmation is always necessary. In the case of an atypical location, clinical or diagnostic indication of a malignant origin and/or in the case of a lack of response to drug therapy, a histological specimen should be acquired immediately.

The differentiation between a primary/idiopathic and a secondary form of fibrosis is essential since the former responds extremely well to immunosuppresive treatment while drug therapy is usually not effective in the case of a secondary form. Important aspects for a more precise classification were presented by Fernando et al. and described again in detail by Brandt et al. [9-11]:

\section{Idiopathic RPF without the presence of cofactors.}

In $75 \%$ of cases, the etiology cannot be determined so that idiopathic fibrosis (IRF) is diagnosed [4].

\section{RPF as a consequence of or in combination with arterial vascular disease}

Aneurysms or arteriosclerosis can be seen in approximately 10$20 \%$ of patients with RPF. The escape of antigens through the weakened media resulting in a local immune reaction with inflammation and subsequent fibrosis has been discussed as the pathogenesis [12].

3. RPF as a consequence of or in combination with an autoimmune disease or vasculitis

A correlation between RPF and autoimmune diseases (e. g. Hashimoto's thyroiditis) has been described by various authors in approx. 10-20\% of cases. Therefore, RPF diagnosis should include investigation of a possible autoimmune origin [12, 13]. An important differential diagnosis from the group of rheumatological diseases is Erdheim-Chester disease (ECD), a rare form of non-Langerhans cell histiocytosis with a poor prognosis in some cases and a high mortality rate. Multisystemic disease typically includes involvement of the long bones, periarterial inflammation primarily of the aorta, and retroperitoneal, usually perirenal, fibrosis. A typical sign on CT is the so-called "hairy kidneys" $[14,15]$.

\section{IgG4-associated RPF}

In recent years there has been a focus on the theory of IgG4-associated retroperitoneal fibrosis which is based on the histological verification of IgG4-positive plasma cell infiltrates. Moreover, elevated serum IgG4 levels are seen in up to $60 \%$ of RPF patients. Therefore, the percentage of IgG4-associated RPF cases among patients otherwise classified as having idiopathic or primary RPF could by higher than previously assumed. $[5,16]$. The most common form of $\mathrm{lgG} 4$-associated disease is type 1 autoimmune pan- 
creatitis. IgG4-associated diseases have the following in common: good response to glucocorticoids usually associated with rapid response of clinical and laboratory parameters. In the case of a lack of response to steroids, tamoxifen, methotrexate (MTX), azathioprine, and cyclophosphamide are available as alternatives [17].

Arora et al. showed that an IgG4-associated disease should be assumed also in the case of negative histology and the presence of an elevated $\mathrm{lgG} 4 /$ total $\mathrm{lgG}$ ratio. They emphasized the fact that the symptoms of patients with and without positive histology do not differ and that the $\mathrm{lgG} /$ total $\mathrm{lgG}$ ratio is the method of choice with the greatest sensitivity in contrast to histology [8].

\section{Drug-induced/toxic RPF}

Smoking, ergotamine derivatives (primarily methysergide), opioid abuse, and exposure to asbestos are risk factors for RPF. Smoking, ANA positivity, and lumbar pain were associated with the recurrence of retroperitoneal fibrosis after initial remission following steroid and/or MTX therapy [18-20].

\section{RPF resulting from radiation or surgery}

Since there is little correlation between this secondary form of RPF and Ormond's disease, it should be classified and treated as radiation fibrosis or scarring [21].

\section{Paraneoplastic/malignant RPF}

It is not yet clear whether this form of secondary RPF is an independent entity or rather represents (pseudo) fibrosis occurring as part of a malignant primary disease (e. g. lymphoma, sarcoma) thus representing a differential diagnosis. However, it has been shown that this form does not respond to drug therapy with immunosuppressants. Therefore, the focus should be on diagnosis, e. g. via biopsy, and treatment of the primary malignant disease [11].

\section{Morphological characteristics}

Radiological imaging plays a central role and is the gold standard in the diagnosis and follow-up of RPF since it helps to determine whether, for example, double-J stents can be removed or a medication can be paused, while the value of many laboratory parameters for treatment monitoring is still unclear [11, 22].

Morphological CT parameters, such as Hounsfield units during the venous and the late phase, and the maximum fibrosis diameter, are considered predictive markers for improvement or worsening of renal function in RPF after drug therapy [23].

\section{Ultrasound}

Ultrasound was considered inadequate. However, newer publications by authors like Kamper et al. have shown that modern ultrasound can be used for follow-up under special circumstances [24]. The images from 35 patients with MRI-confirmed RPF with typical extension were correlated with four standardized ultrasound views (transverse at the level of the renal pedicle, on the level of the aortic bifurcation and transverse through each common iliac artery). Conventional B-mode "tissue harmonic imaging" and the wide-angle function were used. In particular, "tissue harmonic imaging" could adequately visualize the extent of RPF in $94.2 \%$ $(n=33)$ patients and performed significantly better than B-mode imaging.

\section{CT/MRI}

However, CT and MRI were used for imaging in most cases.

On CT, RPF appears as an isodense periaortic mass, typically under the renal pedicle with common iliac extension. As a sign of active RPF, intense contrast enhancement is often seen. Dilatation of the urinary tract can be effectively visualized and tracked. The affected vessels can be sufficiently evaluated (see $>$ Fig. 1, 5).

MRI also allows precise evaluation of the extent of RPF and the associated complications. RPF is seen on non-contrast T1w sequences as a hypointense to isointense mass and in the case of untreated active RPF additionally with a hyperintense signal in T2-weighted sequences and intense contrast enhancement in T1w-fs sequences after IV application of contrast agents containing gadolinium (see $>$ Fig. 3). With high signal intensities and a correspondingly low signal in the ADC map, diffusion-weighted sequences also show typical signal behavior in the case of active RPF [25-27]. In a study including 21 patients, Kamper et al. were able to show a significant decrease in the b800 signal intensity and higher ADC values in DWI [28]. Particularly in patients with limited renal function or in young patients, MRI can provide important information without radiation and potentially also without the use of contrast since DWI sequences are very useful for evaluating acute RPF [27, 29].

\section{Perfusion CT (VPCT)}

Newer methods like perfusion CT (VPCT) can be used to evaluate fibrosis activity in addition to the already identifiable morphological criteria such as extension and density (see - Fig.6). Bier et al. evaluated the role of VPCT for determining inflammatory activity in patients with acute and chronic periaortitis and undergoing immunotherapy. In a study including 35 patients, the parameters of VPCT (blood flow, blood volume, volume transfer constant ktrans, time to peak, mean transit time) in untreated patients correlated significantly with serological markers (CRP, BSG) in relation to disease activity but only correlated weakly in treated patients which emphasizes the value of VPCT for initial diagnosis but leaves the question of its use for follow-up open [30]. A second study including 17 patients undergoing immunosuppressive therapy showed a significant decrease in the parameters "blood flow" and "blood volume" during follow-up via VPCT [31]. Therefore, it seems to effectively show the changes in hemodynamics with respect to inflammation. However, a limitation regarding dynamic CT examination is that it is associated with relevant radiation exposure and therefore should not be routinely used for follow-up in the case of clinical improvement or in patients who are symptom-free.

\section{Hybrid imaging}

Hybrid imaging plays a special role (see $>$ Fig. 2). FDG-PET/CT or FDG-PET/MRI should be considered as an additional or primary modality since hybrid imaging can evaluate both morphological changes and a decrease in inflammation based on the reduced 


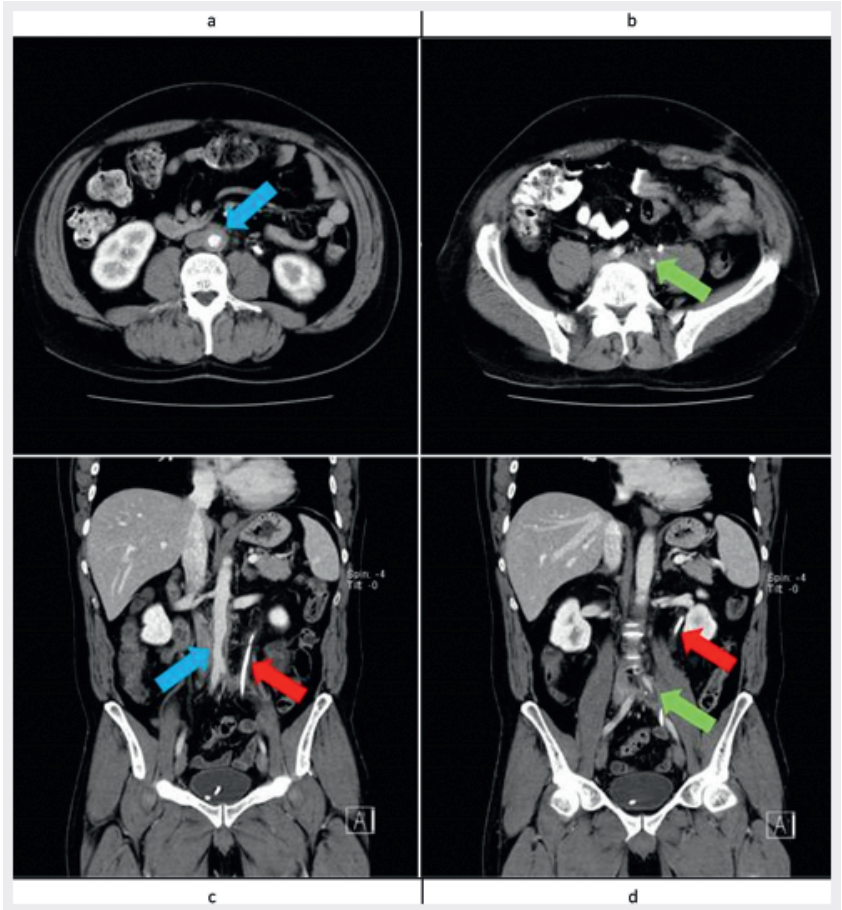

- Fig. 1 CT in arterial (a, b, axial MPRs) and portal venous (c, d, coronal MPRs) contrast medium phase. Classic image of an RPF with iso- to hypodense, retroperitoneal, periaortic mass (blue arrow) under the kidney to the left iliac artery (green arrow). Consecutive compression of the left ureter with urinary congestion, which resulted in ureterolysis and application of a double-J catheter (red arrow).

tracer uptake [32]. Therefore, for example, in a recent monocentric retrospective cohort study, Morin et al. were able to again highlight the value of FDG-PET/CT in primary diagnosis and particularly in follow-up. In their cohort $(n=23)$, all patients showed increased tracer uptake on FDG-PET/CT at the time of diagnosis. In addition, the persistent FDG uptake after treatment with steroids in the second follow-up examination was significantly associated with the recurrence of IRF [33]. Other authors also highlight the importance of hybrid imaging, which allows differentiation, for example, between RPF and lymphoma. In 2017, Fernando et al. included 78 patients with RPF in a prospective study [3]. $0 \%$ of patients with negative $\left[{ }^{18} \mathrm{~F}\right]-\mathrm{FDG}-\mathrm{PET}(\mathrm{n}=24)$ had a malignancy in the subsequent biopsy (negative predictive value $100 \%$ ). All malignant masses were correctly identified and subsequently histologically verified $(n=4)$. The patients with malignant masses exhibited significantly increased tracer uptake (SUV max $\geq 4$ ) and an atypical distribution pattern. FDG-PET/CT was able to show RPF activity in $50 \%$ of patients with unremarkable lab results (19/38). Patients with high activity on FDG-PET/CT responded significantly better to steroid therapy than patients with low or no activity on FDG-PET/CT. An advantage of hybrid imaging that should not be underestimated is the potential to dispense with the use of intravenous contrast agent. Due to RPF and subsequent hydronephrosis, many patients have significantly limited kidney function so that the administration of IV contrast agent is often contraindicat-

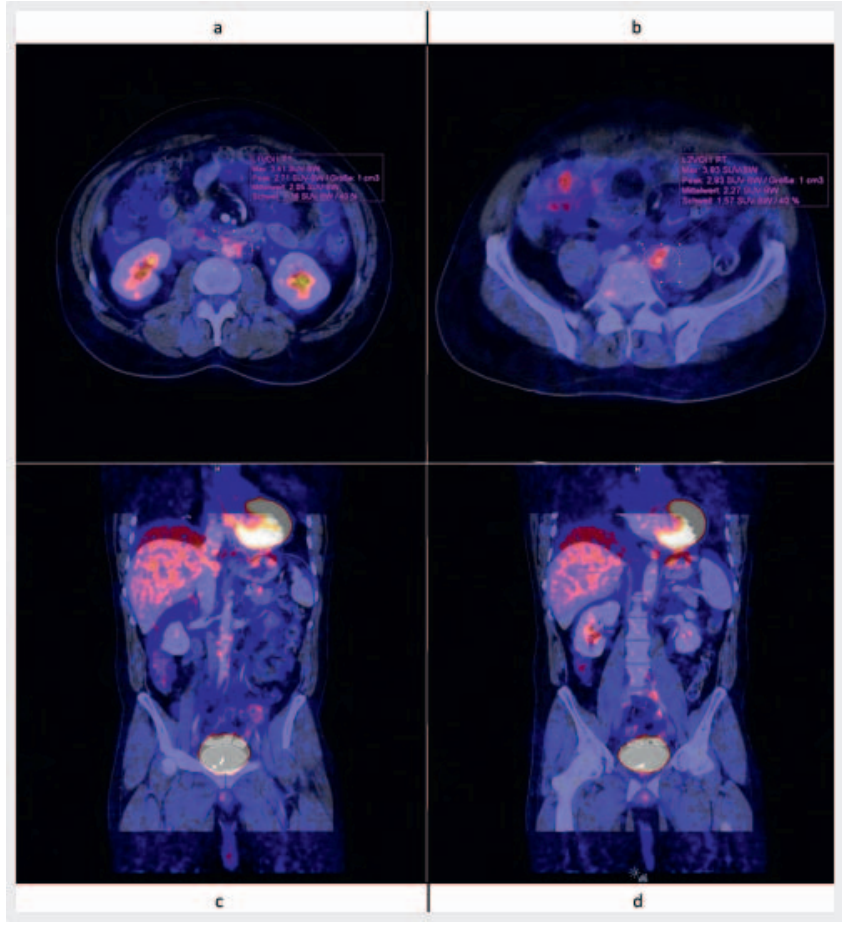

- Fig. 2 [18F]-FDG-PET/CT of the patient described in $>$ Fig. 1. The retroperitoneal mass shows moderate metabolic activity with an SUVmean of 2.1 to 2.3, correlating with a florid RPF (a, b axial MPRs, c, $\mathbf{d}$ coronal MPRs).

ed. In such cases PET/CT can still provide useful information while maintaining good anatomical resolution by applying FDG without IV contrast agent (low-dose CT should not be used exclusively for attenuation correction) [34]. Individual case reports show that it is possible to remove stents or catheters early in the case of a lack of FDG uptake in spite of morphological persistence of RPF [35].

FDG-PET/MRI hybrid imaging is unfortunately not universally available but represents an alternative to $\mathrm{PET} / \mathrm{CT}$ particularly in young patients due to the reduced radiation exposure and to $\mathrm{CT}$ and MRI due to the additional information. In addition to the above-mentioned morphological criteria in T2, T1_fs_contrastenhanced, DWI and ADC sequences, it provides additional functional information as a result of the FDG uptake. Ruhlmann et al. emphasize the higher value of PET parameters compared to MRI parameters with respect to differentiating between treated and untreated patients [27].

Hybrid imaging both with FDG-PET/CT and FDG-PET/MRI therefore plays a special role in RPF imaging since it is highly suited for the quantification and prediction of treatment response, allows corresponding drug therapy adjustments, and allows potentially earlier removal of any foreign objects like stents and catheters. $[3,35]$ 


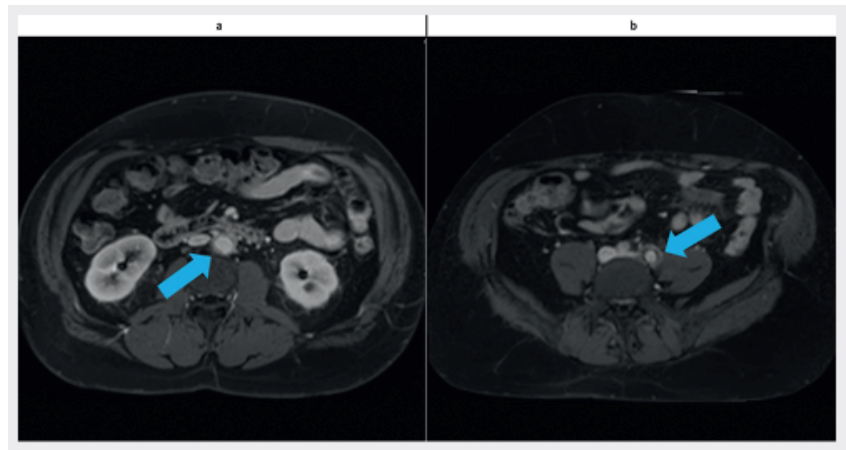

- Fig. 3 MRI of the patient shown in $>$ Fig. 1, 2, four years after initial diagnosis and currently under immunosuppressive therapy (a, b: sequence: T1_fl2d_fs_tra_postKM). The RPF is smaller in size, with contrast medium uptake still present in the marginal area. However, residual activity must be assumed.

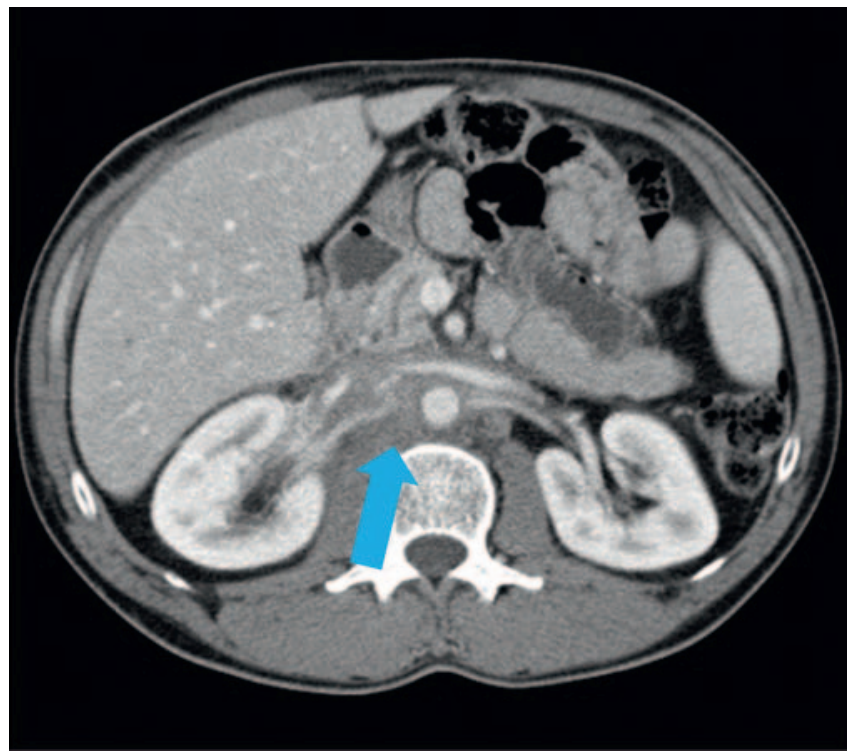

- Fig. 4 Axial CT reconstruction in the portal vein contrast medium phase: patient with histologically confirmed follicular lymphoma after four cycles CHOP. Retroperitoneal mass resembles an RPF, but spreads cranially over the renal pedicle.

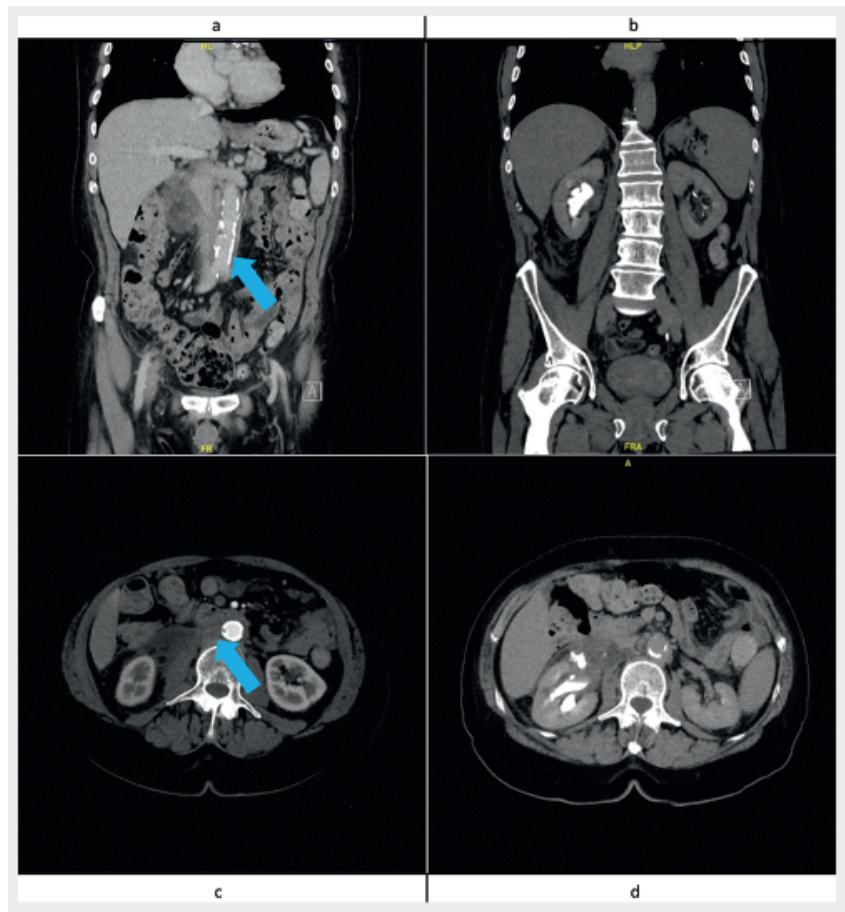

- Fig. 5 Coronal a, b and axial c, d CT reconstructions in the portal vein $\mathbf{a}$, arterial $\mathbf{c}$ and urographic contrast medium phase $\mathbf{b}$, $\mathbf{d}$. One of the most common complications of RPF is compression and stenosis of the ureters. In this example on the right side, with consecutive hydronephrosis on the right, delayed contrast medium excretion and fornix rupture are seen. 
- Table 1 Morphological criteria for RPF.

\begin{tabular}{|c|c|c|}
\hline modality & morphological criteria for RPF & potential indications of malignancy \\
\hline ultrasound & $\begin{array}{l}\text { determination of extent of cancer via "tissue harmonic imaging" in standard } \\
\text { views, extension typically below the renal pedicle }\end{array}$ & \multirow{9}{*}{$\begin{array}{l}\text { extension above the vessels of the renal pedicle } \\
\text { retroaortic extension } \\
\text { pathologically enlarged lymph nodes }\end{array}$} \\
\hline \multirow[t]{4}{*}{ CT } & isodense periaortic mass, typically below the renal pedicle to periiliac & \\
\hline & intense contrast enhancement can indicate active RPF & \\
\hline & urinary retention & \\
\hline & Calcifications as a possible sign of inactive RPF & \\
\hline \multirow[t]{4}{*}{ MRI } & T1w non-contrast hypointense to isointense & \\
\hline & T2 non-contrast hyperintense in the case of activity & \\
\hline & intense contrast enhancement in T1w-fs in the case of activity & \\
\hline & high DWI signal or correspondingly low ADC signal in the case of activity & \\
\hline \multirow[t]{2}{*}{ PET-CT/MRI } & morphological criteria mentioned above & \multirow{2}{*}{$\begin{array}{l}\text { greatly increased (SUVmax } \geq 4 \text { ) or atypically } \\
\text { distributed FDG uptake can indicate } \\
\text { malignancy }\end{array}$} \\
\hline & increased FDG uptake in terms of an increase in metabolic activity & \\
\hline
\end{tabular}

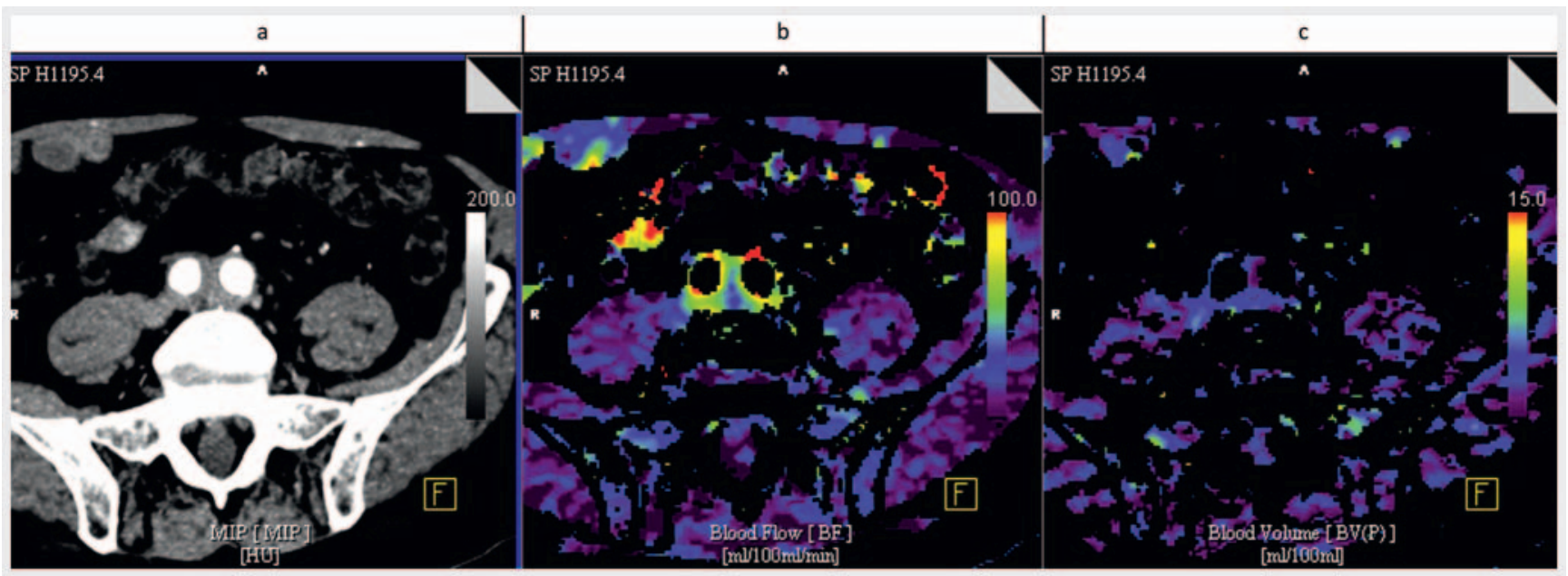

- Fig. 6 Perfusion CT of a patient with active RPF: contrast medium-affine, inhomogeneous hyperperfused tissue proliferation around the infrarenal aorta and the proximal iliac vessels on both sides, matching a highly active RPF (a-c axial MPRs; a: MIP; b: blood flow; c: blood volume).

\section{Summary}

Ormond's disease in terms of primary or idiopathic retroperitoneal fibrosis is a rare but important differential diagnosis in nonspecific back and flank pain. The terminology to be used to differentiate from other forms of fibrotic processes in the retroperitoneum is not clearly defined. Therefore, RPF should be categorized as primary or secondary. The acronym proposed by Fernando et al. RPF ("Right diagnosis"; "Preservation of renal function"; "Freedom from stents, steroids and pain") summarizes the key aspects of the clinical picture of RPF [9]. Radiological imaging by means of ultrasound, CT, MRI, and FDG-PET/CT or FDG$\mathrm{PET} / \mathrm{MRI}$ plays a key role with respect to "right diagnosis" and "freedom from stents, steroids and pain" by allowing noninvasive diagnosis and, in contrast to lab parameters, allowing more reliable follow-up of RPF and any necessary treatment adjustments.
MRI is particularly suitable here as a method for repeated followup examinations due to the lack of radiation. The differentiation of retroperitoneal masses from other differential diagnoses, particularly lymphomas, sarcomas, multiple myeloma, and Erdheim-Chester disease is essential since most patients with RPF have an excellent prognosis with proper treatment.

\section{Conflict of Interest}

The authors declare that they have no conflict of interest. 
[1] Vaglio A, Salvarani C, Buzio C. Retroperitoneal fibrosis. Lancet 2006; 367 : 241-251. doi:10.1016/S0140-6736(06)68035-5

[2] Vogt B, Meier P, Burnier M. Retroperitoneal fibrosis, M. Ormond, periaoartitis, ...? Ther Umsch 2008; 65: 265-268. doi:10.1024/0040-5930.65.5.265

[3] Fernando A, Pattison ], Horsfield C et al. [(18)F]-Fluorodeoxyglucose Positron Emission Tomography in the Diagnosis, Treatment Stratification, and Monitoring of Patients with Retroperitoneal Fibrosis: A Prospective Clinical Study. Eur Urol 2017; 71: 926-933. doi:10.1016/j.eururo.2016.10.046

[4] Swartz RD. Idiopathic retroperitoneal fibrosis: a review of the pathogenesis and approaches to treatment. Am J Kidney Dis 2009; 54: 546553. doi:10.1053/j.ajkd.2009.04.019

[5] Chandna A, Sharma AP, Pareek T et al. IgG4-related Retroperitoneal Fibrosis: An Emerging Masquerader With a Sinister Presentation. Urology 2019; 133: 16-20. doi:10.1016/j.urology.2019.06.007

[6] Cristian S, Cristian M, Cristian P et al. Management of idiopathic retroperitoneal fibrosis from the urologist's perspective. Ther Adv Urol 2015; 7: 85-99. doi:10.1177/1756287214565637

[7] Zhang S, Chen M, Li CM et al. Differentiation of Lymphoma Presenting as Retroperitoneal Mass and Retroperitoneal Fibrosis: Evaluation with Multidetector-row Computed Tomography. Chin Med J (Engl) 2017; 130: 691-697. doi:10.4103/0366-6999.201606

[8] Arora K, Rivera M, Ting DT et al. The histological diagnosis of IgG4-related disease on small biopsies: challenges and pitfalls. Histopathology 2019; 74: 688-698. doi:10.1111/his.13787

[9] Fernando A, Pattison J, Horsfield C et al. A lot of questions (and a few answers ...) in retroperitoneal fibrosis. BJU Int 2016; 117: 16-19. doi:10.1111/bju.13061

[10] Takahashi Y, Matsushima H, Mori T et al. Retroperitoneal fibrosis after chemo-radiotherapy for cervical cancer: A case report. J Obstet Gynaecol Res 2019; 45: 938-941. doi:10.1111/jog.13898

[11] Brandt AS, Dreger NM, Muller E et al. New (and old) aspects of retroperitoneal fibrosis. Urologe A 2017; 56: 887-894. doi:10.1007/ s00120-017-0428-y

[12] Brandt AS, Kamper L, Kukuk S et al. Associated findings and complications of retroperitoneal fibrosis in 204 patients: results of a urological registry. J Urol 2011; 185: 526-531. doi:10.1016/j.juro.2010.09.105

[13] Ceresini G, Urban ML, Corradi D et al. Association between idiopathic retroperitoneal fibrosis and autoimmune thyroiditis: a case-control study. Autoimmun Rev 2015; 14: 16-22. doi:10.1016/ j.autrev.2014.08.006

[14] Van Keerberghen CA, Harrouk A, Leone L. A new role for fluorine-18fluorodeoxyglucose positron-emission tomography/computed tomography in Erdheim-Chester disease. World J Nucl Med 2019; 18: 201-203. doi:10.4103/wjnm.WJNM_57_18

[15] Knitza J, Kampylafka E, Wacker J et al. Erdheim-Chester disease: An important differential diagnosis and its main symptoms. Z Rheumatol 2019; 78: 66-71. doi:10.1007/s00393-018-0566-7

[16] Lian L, Wang C, Tian JL. IgG4-related retroperitoneal fibrosis: a newly characterized disease. Int J Rheum Dis 2016; 19: 1049-1055. doi:10.1111/1756-185X.12863

[17] Zen Y, Onodera M, Inoue D et al. Retroperitoneal fibrosis: a clinicopathologic study with respect to immunoglobulin G4. Am J Surg Pathol 2009; 33: 1833-1839. doi:10.1097/pas.0b013e3181b72882

[18] Mohammadzadeh Rezaei MA, Akhavan Rezayat A, Ardalan S. Retroperitoneal Fibrosis Due to Opium Abuse: A Case Series and Literature Review. Nephrourol Mon 2016; 8: e39788. doi:10.5812/numonthly.39788

[19] Goldoni M, Bonini S, Urban ML et al. Asbestos and smoking as risk factors for idiopathic retroperitoneal fibrosis: a case-control study. Ann Intern Med 2014; 161: 181-188. doi:10.7326/M13-2648

[20] Moriconi D, Giannese D, Capecchi R et al. Risk factors for relapse and long-term outcome of idiopathic retroperitoneal fibrosis. Clin Exp Nephrol 2019; 23: 1147-1153. doi:10.1007/s10157-019-01759-w

[21] Kocot A, Riedmiller $H$. Treatment of long-term radiation injuries in the urinary tract. Urologe A 2015; 54: 1765-1771. doi:10.1007| s00120-015-4011-0

[22] Bertagna F, Treglia G, Leccisotti L et al. [(1)(8)F]FDG-PET/CT in patients affected by retroperitoneal fibrosis: a bicentric experience. Jpn J Radiol 2012; 30: 415-421. doi:10.1007/s11604-012-0066-7

[23] Gao L, Wang H, Xu Y et al. Computed tomography parameters can be used as predictive markers for the improvement of renal function in patients with retroperitoneal fibrosis. Clin Exp Rheumatol 2015; 33: 871-876

[24] Kamper L, Brandt AS, Ekamp H et al. The potential role of modern US in the follow-up of patients with retroperitoneal fibrosis. Diagn Interv Radiol 2014; 20: 3-8. doi:10.5152/dir.2013.13132

[25] Kamper L, Brandt AS, Scharwachter $C$ et al. MR evaluation of retroperitoneal fibrosis. Rofo 2011; 183: 721-726. doi:10.1055/s-0031-1273450

[26] Burn PR, Singh S, Barbar $S$ et al. Role of gadolinium-enhanced magnetic resonance imaging in retroperitoneal fibrosis. Can Assoc Radiol J 2002; 53: $168-170$

[27] Ruhlmann V, Poeppel TD, Brandt AS et al. (18)F-FDG PET/MRI evaluation of retroperitoneal fibrosis: a simultaneous multiparametric approach for diagnosing active disease. Eur J Nucl Med Mol Imaging 2016; 43: 16461652. doi:10.1007/s00259-016-3351-3

[28] Kamper L, Haage P, Brandt AS et al. Diffusion-weighted MRI in the follow-up of chronic periaortitis. Br J Radiol 2015; 88: 20150145. doi:10.1259/bjr.20150145

[29] Kamper L, Dreger NM, Brandt AS et al. Diffusion-weighted MRI and PET-CT in the follow up of chronic periaortitis. Int J Cardiovasc Imaging 2018; 34: 1779-1785. doi:10.1007/s10554-018-1395-0

[30] Bier G, Henes ], Eulenbruch $C$ et al. Perfusion-based assessment of disease activity in untreated and treated patients with aortitis and chronic periaortitis: correlation with CT morphological, clinical and serological data. Br J Radiol 2015; 88: 20150526. doi:10.1259/bjr.20150526

[31] Bier G, Kurucay M, Henes J et al. Monitoring Disease Activity in Patients with Aortitis and Chronic Periaortitis Undergoing Immunosuppressive Therapy by Perfusion CT. Acad Radiol 2017; 24: 470-477. doi:10.1016/ j.acra.2016.10.013

[32] Wiggermann P, Aust D, Stroszczynski C et al. Follow-up of FDG-PET/CT findings in retroperitoneal fibrosis associated with abdominal aortic aneurysm. Nuklearmedizin 2011; 50: N21-N22

[33] Morin G, Mageau A, Benali K et al. Persistent FDG/PET CT uptake in idiopathic retroperitoneal fibrosis helps identifying patients at a higher risk for relapse. Eur ] Intern Med 2019; 62: 67-71. doi:10.1016/ j.ejim.2019.01.019

[34] Talati SJ, Abghari R, Kochkodan JJ et al. Use of Ga-67 imaging in diagnosis and follow-up after steroid treatment of retroperitoneal fibrosis. Clin Nucl Med 1995; 20: 995-997. doi:10.1097/00003072-19951100000012

[35] Piccoli GB, Consiglio V, Arena V et al. Positron emission tomography as a tool for the 'tailored' management of retroperitoneal fibrosis: a nephrourological experience. Nephrol Dial Transplant 2010; 25: 2603-2610. doi:10.1093/ndt/gfq051

[36] Koike Y, Matsui S, Takase K et al. CT-Guided Percutaneous Needle Biopsy in Patients with Suspected Retroperitoneal Fibrosis: A Retrospective Cohort Study. Cardiovasc Intervent Radiol 2019; 42: 1434-1440. doi:10.1007/s00270-019-02266-x 
[37] Loricera J, Blanco R, Castaneda S et al. Tocilizumab in refractory aortitis: study on 16 patients and literature review. Clin Exp Rheumatol 2014; 32 : S79-S89

[38] Perrotta FM, Fici M, Guerra G et al. Chronic periaortitis with retroperitoneal fibrosis successfully treated with first line tocilizumab monotherapy: a case report. Clin Exp Rheumatol 2017; 35 (Suppl. 103): 226-227

[39] Dedinska I, Svetlik D, Adamicova K et al. Treatment of Ormond Disease and Idiopathic Membranous Glomerulonephritis by using Rituximab. Iran J Kidney Dis 2016; 10: 332-335

[40] Catanoso MG, Spaggiari L, Magnani L et al. Efficacy of infliximab in a patient with refractory idiopathic retroperitoneal fibrosis. Clin Exp Rheumatol 2012; 30: 776-778 